wherein morphia is manufactured to this day by a process originally based on that devised by him more than a century ago.

"Alison, W. P., Proc. Roy. Soc. Edin., 4 (1857-62). "An Account of the Iife and Labours of Dr. Wm. Gregory".

2 Scotsman, Edinburgh (May 1, 1858).

s Scotsman, Edinburgh (March 3, 1938). (Tercentenary notice to James Gregory, 1638-1675.)

4 Proc. Chem. Soc., 12, 172 (1859).

s Gregory, Wm., "Outline of Chemistry" (London: Taylor and wory, edition 1847 re-issue in two volumes, Walton, 1845) ; Chemistry" (T. Gerding, Brunswick, 1854).

- Gregory, Wm., "On the State of the Schools of Chemistry in the United Kingdom" (London: Taylor and Walton, 1842) Pamphlet 102 , Library of the Chemical Society, London.

7 Royal Society of Catalogue of Scientific Papers (1869), Volume 3, $1800-1863$.

8 Gregory, Wm., Proc. Chem. Soc., 7-8 (1841-43).

- Gregory, Wm., "Letters to a Candid Inquirer on Animal Magnetism" (London: Taylor, Walton and Maberley, 1851).

10 "The Life of Sir Robert Christison, Bart.", edited by his sons (William Blackwood and Sons, 1886).

11 Gregory, Wm., Proc. Roy. Soc. Edin., 2, 316 (1844-1850).

12 Howard, D. Xi., Yearb. Pharm., 347 (1926).

${ }^{13}$ Ind, and Eng. Chem., 4, 364 (1912).

14 Grier, James, "A History of Pharmacy" (London: Pharmareutical Press, 1937).

1s Young, John, "On the Preparation of Opium in Great Britain", Edin. Phil. .J., 1, 258 (1819).

16 Young, John, "A Method of Cultivating Papaver Somniferum in Great Britain and the Preparation of Opium from it", Trans. Soc. Arts, 37 (1819); 38 (1.820).

17 Gregory, Wm., Edin. Med. and Surg. J., 35, 331 (1831).

18 Lancet, 806 (1838-39).

${ }^{10}$ Wood, Alexander, Edin. Med. and Surg. Rev., 82, 265 (1855).

BIBLIOGRAPHY :

“The Academic Gregories", Agnes Grainger Stewart (Edinburgh and London: Oliphant, Anderson and Forries, 1901).

“Franz Anton Mesmer", Margaret Goldsmith (London: Arthur Baker,

Ltd., 1934).
"History of Scottish Medicine to 1860", J. D. Comrie (Wellcome Cox, 1927).

\section{CREEP OF METALS}

$\mathrm{A}^{\mathrm{T}}$

T a conference organised by Sir Charles Darwin, and held at the Royal Society on February 5, opportunity was given for physicists, engineers and metallurgists interested in the 'creөp', or continuous slow deformation under load, of metals to compare their findings and express their several points of view.

It is well known that when a metal 'creeps' under constant temperature and load, the rate of extension at first decreases progressively, then remains constant for a period, and afterwards increases. Prof. E. N. da C. Andrade emphasized the complex nature of the processes involved, but maintained that for all pure metals below their recrystallization temperature range, the use of a constant stress in place of a constant load results in the elimination of the final increase of rate of strain, the creep then taking place according to the relation

$$
l_{t}=l_{0}\left(1+\beta t^{1 / 3}\right) e^{k t},
$$

where $l_{t}$ is the original length, $l_{0}$ the length -after time $t$, and $\beta$ and $k$ are constants. The constant $\beta$ never rises above a certain value, and represents a type of transient flow associated with rotation of the crystals, while $k$ represents a uniform rate of flow due to movement along the glide planes. The $k$ flow is governed by an activation energy, analogous to that determining the rate of viscous flow of Newtonian liquids, so that, for varying temperatures, the rate of flow is proportional to $e^{-E / k t}$ (where $E$ is the activation energy); but it differs from the viscous flow of liquids in that the activation energy is a function of the applied stress. In the case of single crystals, the activation energy takes the form

$$
E=A\left(S_{0}-g S\right)^{2},
$$

where $S_{0}$ is the critical shear stress necessary to cause glide in glide plane, $S$ is the applied stress and $g$ is a stress concentration factor. Consequently,

$$
\text { creөp-rate }=C \exp .\left[-\frac{A}{k T}\left(S_{0}-g S\right)^{2}\right] .
$$

This expression, which originated in a modification by Orowan of the theory proposed by Becker, has been confirmed for the creep of single crystals in cases where the stresses are not too big. The necessary activation energy is regarded as being supplied by thermal fluctuations and spans the gap between the stress necessary to cause $\operatorname{slip} S_{0}$ and the stress $g S$ present at points of stress concentration in the crystal.

Prof. Andrade emphasized the point that, with polycrystalline wires or rods subjected to tension at constant load and high temperature, the rate of flow may increase for two quite different reasons : (a) increase of stress, and (b) slow recrystallization processes. Flow (a) will take place with all metals if the extensions are large, say of the order 5 per cent or more : on the other hand, flow (b) will take place with certain metals, and not with others, and may occur even where the extension is a fraction of 1 per cent, and so the stress tolerably constant. The form of the curve of extension against time is similar in case $a$ at many per cent, and in case $b$ at much smaller extensions, although the physical processes are quite different in the two cases; this has led to much confusion, especially as to the necessity of maintaining constant stress. In case $b$ the material at the end of the test is not the same as at the beginning.

Mr. H. J. Tapsell, after describing the methods employed at the National Physical Laboratory in testing the creep resistance of engineering materials, directed attention to the extreme slowness (of the order of $10^{-8}$ strain per hour) of the changes in which the testing engineer is interested, and showed the difficulty of expressing the behaviour of engineering materials in simple terms by quoting examples of unpredictable changes in creep-rate which may occur after long periods of test. These render long-time tests a practical necessity, and the problem of predicting safe ranges of stress for engineering components which must give satisfactory service for periods of many years is one of real difficulty. How ever, a set procedure of extrapolation from the behaviour during short periods at temperatures rather above the service temperature, combined with the judicious use of empirical formulæ for the variation of creep-rate with stress and time, often enables a practical solution to be found. The practical efficacy of these methods was confirmed later in the discussion by Dr. R. W. Bailey.

Provided tests are limited to the early stage of decreasing creep-rate, simple relationships of the type :

Creep-rate $=A \quad$ (stress) $^{n} \quad$ (at constant temperature and time) Creep-rate $=B$ (time) $-p$ (at constant temperature and stress)

appear to hold for a variety of materials, and creep curves in tension, torsion and combinations of tension and torsion have the same geometric form. When the 
test piece is unloaded, a slow recovery of dimensions follows the immediate elastic contraction, and is thought to be attributable to the strain concentrations between individual crystals during creep.

Dr. E. Orowan suggested a new theoretical approach to the problem of creep and of plastic flow in general. Sudden application of a stress produces a sudden plastic deformation, followed by creep at a rapidly diminishing rate. At the end of the sudden deformation, the resistance of the material to plastic deformation (its yield stress) is just sufficient to balance the applied stress if this is not helped by thermal stress fluctuations; the activation energy for creep at this moment, therefore, is vanishingly small. As creep proceeds, however, the yield stress rises owing to strain hardening, while the applied stress is kept constant. For relatively small creep strains, the rise of the yield stress is proportional to the creep strain $\gamma$, and so the additional stress that has to be supplied by thermal fluctuations in order to produce creep rises proportionally to the creep strain. If, as in the Becker theory, the activation energy is assumed to be proportional to the square of the activation stress, the frequency of local creep processes is proportional to

$$
\text { exp. }-n \gamma^{2} / T
$$

where $n$ is a constant. If it is assumed that, with polycrystalline metals and single crystals of cubic metals, the contribution of a successful stress fluctuation to the macroscopic creep strain is inversely proportional to $\gamma^{2}$, the creep-rate is given by

$$
\frac{d \gamma}{d t}=\frac{\text { const. }}{\gamma^{2}} \text { exp. }-n \gamma^{2} / T \text {. }
$$

Measurements of creep curves by J. Los in the Cavendish Laboratory were found to be represented by this formula with a high degree of accuracy.

If there is a noticeable recovery (thermal softening), the decrease of the yield stress due to this factor must be subtracted from the activation stress ; after prolonged creep at not too low temperatures, this may become a dominating factor, and then the above formula goes over into the expression resulting from the well-known recovery theory of creep. The simple recovery theory, however, is unable to account for the slowing down of the creep in the initial stage. In its original form, the Becker theory is equally unable to explain the slowing down of the creep; if strain hardening is introduced into the BeckerOrowan formula, other difficulties arise from the circumstance that, according to experiments, the rate of creep is not uniquely determined by stress, strain and temperature.

Dr. Orowan mentioned that, according to his new theory, some characteristic features would be due to the circumstance that creep is distinguished from other known reactions by the increase of the activation energy during the reaction. The presence of vanishingly small activation energies at the beginning of the creep have received experimental confirmation by the observed creep of cadmium and zinc crystals at very low temperatures (down to $1 \cdot 2^{\circ}$ abs.).

A different and novel method of regarding the strain hardening was contributed by Dr. A. McCance, who related the hardening to the increase in volume which accompanies plastic deformation. By assuming that each element of slip along the glide plane is accompanied by a proportional separation at right angles to the glide plane, he showed that for an ordinary tensile test the stress should be related to strain by an expression of the form

$$
S=A e^{-\alpha x} \sinh \beta x,
$$

where $S$ is the stress, $x$ the strain and $A$ and $\alpha$ and $\beta$ are constants; he demonstrated that if the contraction of area be used as the measure of strain, a value of $\beta$ can be chosen for a variety of materials such that $\log S / \sinh \beta x$ gives a linear plot against $x$. The further assumption that when the metal is subject to continuous creep, the velocity of shear is pro. portional to the difference between the applied stress $S$ and a suitably defined limiting shear stress $S_{0}$ led to an equation of the form

$$
x=2 x_{0} e^{-\alpha} \sinh \sqrt{\alpha^{2}+\beta\left(S-\overline{S_{0}}\right)} \cdot t,
$$

where $x$ is the extension after a time $t, x_{0}$ is the total extension which takes place after an infinite time under the limiting stress $S_{0}$, and $\alpha$ and $\beta$ are constants. It was then shown that change in the value of $\left(S-S_{0}\right)$ in this equation reproduces qualitatively the effect of changes of stress on the form of the creep curve, and that for certain creep curves values of $\sqrt{\alpha^{2}+\beta\left(S-S_{0}\right)}$ could be chosen such that

$$
x
$$

$\overline{\log \sinh } \sqrt{\sqrt{\alpha^{2}+\beta\left(S-S_{0}\right)} \cdot t}$ gives a linear plot against $t$. Attention was directed to the possibility of deducing the value of $S_{0}$ from a few short-time creep tests, and of forecasting the conditions under which the inflexion in the time-strain curve which precedes fracture would occur. These predictions would naturally be subject to the condition that the metallurgical structure of the material remains constant over the times under consideration.

The influence of metallurgical structure was dealt with briefly by Dr. N. P. Allen, who, after pointing out the increasing need for the development of metals capable of serving at much higher temperatures than have hitherto been used, described the phenomena of which advantage is taken in producing creep. resistant alloys. Increasing the grain-size of the material improves the creep resistance, but is attended by certain disadvantages, and reliance is placed more commonly on the addition of elements which raise the softening temperature of the material, or produce precipitation-hardening effects. There is a close correlation, which probably is significant in relation to the theory of creep, between the effect of added elements on the creep resistance of metals, and the effect of the same elements upon the softening temperature, that is, the temperature at which the additional hardness produced by cold deformation of the metal is removed. The addition of elements which raise the softening temperature, combined with the judicious use of work hardening, is an effective method of improving creep resistance. Precipitation hardening can also be effective, but the relation between the hardening process and the improvement of creep resistance requires further study. Not every alloy which hardens well has good creep resistance ; and, in alloys capable of high creep resistance, the heat treatments which produce the greatest hardness do not necessarily produce the best creep resistance. The compositions and heat treat. ments which give the best results are much influenced by the service temperature, and the form of the solubility curve of the precipitated constituent has an important bearing on the behaviour of the alloy. The creep resistance of certain alloys is also profoundly influenced by small additions made during the deoxidation process, and these additions need to be carefully controlled if uniform results are to be 
obtained. Explanations of these phenomena, which are technically extremely important, are in many cases lacking.

The general discussion was opened by Sir Lawrence Bragg, and divided itself broadly into suggestions and speculations on the part of those who wished to reduce the creep phenomena to simplicity, and warnings-most of them from the engineers and metal lurgists-concerning their essential and extreme complexity. In the first class, Dr. McCance's contribution has already been described. Sir Lawrence Bragg suggested reasons for Dr. Orowan's assumption that the magnitude of the movement accompanying each slip is inversely proportional to the square of the preceding strain, and referred to the possibility of a type of movement in the grain boundaries which is not accompanied by any increase of the resistance to further movement.

Many examples of the unexpected influence of certain factors on the creep strength of metals arose. Dr. L. B. Pfeil described the tenfold superiority of cold-drawn sintered platinum over similarly drawn cast platinum, which superiority, however, is displayed only if the sintering is not carried out at too high a temperature. Dr. C. J. Smithells directed attention to the marked influence of impurities in tungsten and nickel chromium alloys at high temperatures, which led him to think that the grain boundary at high temperatures is a seat of peculiar mobility ; and Mr. E. H. Bucknall to the superior creep strength of the so-called 'intermediate structure' of alloy steels, which is usually regarded as somewhat inferior in general mechanical properties to the conventional quenched and tempered structure. Mr. D. A. Oliver showed how the creep strength of certain alloys is significantly influenced by the temperature at which the final stage of work hardening is carried out. Dr. R. W. Bailey referred to the importance of progressive softening of the metal during the creep test, which proceeds more quickly in heavily cold-worked than in lightly cold-worked material, and accounts for the inferiority of cold-worked material at high temperatures. Sir Charles Darwin questioned whether the creep is in fact continuous with time, eliciting the information that discontinuous creep has been observed on several occasions ; and Mr. C. G. Conway referred to the important point that the imminence of fracture cannot be deduced from the rate of creep.

No summing up of the discussion was attempted, but the writer might venture one general impression - that of the unreliability of the too exclusively mathematical approach. It is simple to arrive at a formula which shall predict, with fair accuracy, an observed behaviour; but it is another matter to demonstrate that the processes assumed in the derivation of the formula are actually going on in the metal.

N. P. AlLEN.

\section{O BITUARIES}

\section{Prof. V. Dolejšek}

THE death of Prof. V. Dolejšek in January 1945, after much suffering, deprived Czechoslovakia of one of its most outstanding men of science. Dolejšek was a distinguished experimental physicist and X-ray spectroscopist. Born on February 20, 1895, in Prague, he studied at the Charles University, where he obtained his doctorate in 1919. In 1922, he worked with Prof. Manne Siegbahn in Lund, and later with Prof. Paschen in Tübingen. In 1928 he was made a temporary professor and in 1935 a permanent professor of experimental physics at the Charles University, Prague.

Dolejšek's scientific career started in the laboratories of Prof. Siegbahn. As a result of his extraordinary experimental skill, he attained an accuracy of measurement of X-ray wave-lengths which has scarcely been surpassed by more elaborate modern methods. In 1922 he discovered the first lines belonging to the $N$ series of uranium and thorium, and later two lines of bismuth. This important discovery contributed substantially to the development of the Rutherford-Bohr-Sommerfeld theory of atomic structure. This work is noteworthy for its experimental difficulties. The lines were measured in the longwave region 8-13 A., whereas the longest wavelengths previously measured were about 5-7 A.

Dolejšek realized that Moseley's law is only approximately valid and that there exist fluctuations in the function connecting atomic number and frequency of X-ray absorption edges and emission lines. $\mathrm{He}$ discovered that these fluctuations have the same periodicity as the periodic system of the elements, and he deduced very accurate empirical laws for the deviations. He also studied the dependence of these fluctuations on the valency, and discovered a law which is the only general law known to be valid in the intricate system of relationships between all of the relevant factors. He also studied the fine structure of the absorption edges and thus verified the theory of Kronig.

These investigations required many measurements of X-ray lines, and several of his papers are concerned with new discoveries and precision measurements of diagram and non-diagram X-ray lines. The highest accuracy was always attained by comparatively simple means. The long-wave region of $\mathrm{X}$-rays was always Dolejšek's favourite, and he designed and constructed an extremely efficient high-intensity $\mathrm{X}$-ray gas tube for very low voltages of the order of a thousand volts, in conjunction with a vacuum spectrograph, which was used to explore this inaccessible region between X-rays and ultra-violet rays. He employed either plane or concave gratings used at a total reflexion angle, or suitable organic or inorganic crystals. A new focusing method with a bent crystal was devised in his laboratories, which has a very high resolving power and yet requires short exposures. This enabled him to measure many spectra of solid elements and of gases, and to contribute further to our knowledge of the $N$-series. In addition, he studied the resolving power of gratings and crystals, the reflecting power of crystals and their surface structure, penetration of X-rays into crystals and many other related problems.

Later Dolejšek extended his spectroscopic work into optics. Optical absorption spectra of rare earths in solutions were studied by means of a powerful hydrogen tube, and the solvent effect investigated. He demonstrated thus that the optical electrons of rare earths are $4 f$ electrons inside the ions. His success in spectroscopy was largely due to his great knowledge of vacuum technique, for which he developed powerful condensation pumps working with solid paraffin, oil manometers for measurement of very low pressures, variable precision gas leaks and many other devices. His knowledge was also of greatest value to the industry. He collaborated with the Skoda Works on many probleris, such as vacuum 